Onkologie 36 | 5 | 13

\section{Ambulante spezialfachärztliche Versorgung (ASV): Was bedeutet das für die onkologische Versorgung?}

GlaxoSmithKline lud am 6. März zur Fachtagung «Brennpunkt ambulante spezialfachärztliche Versorgung: Kooperation statt Konfrontation» nach München ein. Dabei diskutierten Experten unterschiedlicher Disziplinen die Bedeutung der neuen Versorgungssäule für die Onkologie.

Es gab bereits in der Vergangenheit zahlreiche Reformversuche, die starre Trennung der Sektorengrenzen in Deutschland zu überwinden, indem beispielsweise Krankenhäuser für ambulante Leistungen geöffnet wurden. Durch die Neuordnung des $§ 116 b$ SGB V in Form der ASV ist nun erstmals ein Versorgungsbereich unter gleichen Voraussetzungen sowohl für Krankenhausärzte als auch für niedergelassene Vertragsärzte vorgesehen. Der Anfang soll mit onkologischen Therapien erfolgen. Der Über-
Palliative mCRC-KRAS-wt-Patienten:

30 Monate medianes Gesamtüberleben dank intensiver Erstlinientherapie mit Cetuximab + FOLFIRI

Bei Patienten mit metastasiertem Kolorektalkarzinom (mCRC) und KRAS-Wildtyp kann eine intensive Erstlinientherapie bestehend aus Standardchemotherapie und dem EGFR-Antikörper Cetuximab Vorteile bringen. Prof. Dirk Arnold, Freiburg, erläuterte auf der DGHO-Jahrestagung im Oktober 2012 den Stellenwert der Therapieoption unter anderem anhand der Erbitux ${ }^{\circledR}$ Zulassungsstudie CRYSTAL: Insgesamt 1198 therapienaive Patienten erhielten im Verhältnis 1:1 randomisiert entweder eine Behandlung mit einer Standardchemotherapie (FOLFIRI) oder eine Kombination mit Cetuximab. Im Vergleich zur alleinigen Chemotherapie ermöglichte die versorgung und dem von Niedergelassenen befürchteten Wettbewerb mit Kliniken um Patienten in Ballungsräumen stehen strukturschwache Gebiete gegenüber, in denen eine wohnortnahe onkologische Grundversorgung oft gar nicht geleistet werden kann. Mit der ASV ist im Bereich Onkologie jedoch eine intersektorale Kooperation zwischen den Leistungserbringern verpflichtend vorgesehen.

Johannes Singhammer, MdB, der selbst aktiv an der Entwicklung des Gesetzes beteiligt war, hob hervor, dass die komplexen Vorgänge bei schwerwiegenden Erkrankungen vereinfacht werden sollen. Dies wurde allerdings vom bayerischen KV-Vertreter Martin Degenhardt angezweifelt. Er sieht große verwaltungstechnische Hürden in der Umsetzung, insbesondere was die Leitungsvergütung und Honorarverteilung anbetrifft. Wie PD Dr. Stephan Schmitz, Vorsitzender des Berufsverbandes der Niedergelassenen Hämatologen und Onkologen in Deutschland e.V. (BNHO) ausführte, wird der dem Gesetz zugrunde liegende Netzwerkgedanke zwischen onkologischer Praxis und Klinik vielerorts längst ohne gesetzliches Regelwerk umgesetzt. Dort, wo es z.B. aus persönlichen Gründen nicht funktioniere, würde auch ein gesetzlicher Zwang keine Zusammenarbeit herbeiführen.

Dr. Petra Ortner, München

Quelle

Tagung der GlaxoSmithKline GmbH \& Co KG «Brennpunkt ambulante spezialfachärztliche Versorgung: Kooperation stat Konfrontation» am 6. März 2013 in München

Weitere Informationen bei GlaxoSmithKline GmbH \& Co. KG Andreas Krause andreas.x.krause@gsk.com
Zugabe des Antikörpers in der retrospektiven Analyse der 666 KRAS-wt-Patienten eine signifikante Verbesserung im Gesamtansprechen (ORR: 57,3 vs. $39,7 \%$; p < 0,001) und eine signifikante Verbesserung des medianen Gesamtüberlebens (OS: 23,5 vs. 20,0 Monate; $\mathrm{p}=0,0093$ ). Die Tumorreduktion führte zu einer Verringerung der Symptome und bot die Chance auf Resektion.

Eine retrospektive Subgruppenanalyse der CRYSTAL-Studie zeigte außerdem, dass die Kombination FOLFIRI + Cetuximab eine maximale Symptomlinderung nach bereits 8 Wochen zeigte - im Vergleich zu 16 Wochen bei FOLFIRI alleine. Das Erreichen einer frühen Tumorschrumpfung ( $\geq 20 \%$ innerhalb von 8 Wochen) habe neben dem direkten klinischen Nutzen auch einen hohen prognostischen Stellenwert, so Arnold. Denn im Vergleich zu Patienten ohne frühe Tumorreduktion um $\geq 20 \%$ profitierten Patienten mit früher Tumorschrumpfung von einem im Durchschnitt 11,4 Monate längeren Gesamtüberleben (30,0 vs. 18,6 Monate; $\mathrm{p}<0,001)$. Fast zwei Drittel $(62 \%)$ der mit der Cetuximab-Kombination behandelten KRAS-wt-Patienten erreichten eine frühe Tumorschrumpfung $\geq 20 \%$, unter FOLFIRI allein war dies nur bei der Hälfte (49\%) der Fall. Der Einsatz des Biomarkers KRAS sowie des klinischen Markers «frühe Tumorschrumpfung» ermöglicht somit eine frühe Prognoseabschätzung.

Literaturangaben und weitere

Informationen bei

Merck Serono GmbH

Yvonne Weißhuhn

Alsfelder Straße 17

64289 Darmstadt

yvonne.weisshuhn@external.merckgroup.com

\title{
PharmaTicker+++ PharmaTicker+++ PharmaTicker+++ PharmaTicker+++
}

Amgen. Die neu gestartete Website www.lebenmit-knochenmetastasen.de bietet Krebspatienten, ihren Angehörigen und anderen Interessierten fundierte Informationen über das Leben mit dieser Erkrankung. Hintergrundtexte über Knochen, Metastasen und die Chancen einer Behandlung sowie Tipps mit hohem Praxisbezug und ein Erfahrungsbericht sollen den Betroffenen helfen, sich besser auf ihre Krankheitssituation einzustellen.

Amgen $\mathrm{GmbH}$

Fulvia Kipper

Fulvia.kipper@amgen.com
Roche. In ihren aktuellen Leitlinien empfiehlt die Kommission Mamma der AGO (www.ago-online. de) den neu zugelassenen Antikörper Perjeta ${ }^{\circledR}$ (Pertuzumab) in Kombination mit Herceptin ${ }^{\circledR}$ (Trastuzumab Roche) und Docetaxel als neuen 1st-Line-Standard beim metastasierten HER2+ Mamma-Ca (++). Zudem spricht sie sich für die Anwendung von T-DM1 im Rahmen klinischer Studien bei Trastuzumab-vorbehandelten Patientinnen aus ( + ).

Roche Pharma AG

Ulla Satzger

ulla.satzger@roche.com
AstraZeneca. Die Europäische ArzneimittelAgentur hat die Zusammenfassung der Produkteigenschaften (SmPC) von Faslodex ${ }^{\circledR}$ (Fulvestrant) $500 \mathrm{mg}$ Injektionslösung aktualisiert und neue Daten zum Gesamtüberleben von Frauen mit hormonrezeptor-positivem, lokal fortgeschrittenem oder metastasiertem Mammakarzinom ergänzt. Die abschließende Analyse des Gesamtüberlebens aus der CONFIRM-Studie wurde jetzt auf der 13. Internationalen Brustkrebskonferenz in St. Gallen vorgestellt.

AstraZeneca GmbH

Florian Dieckmann

florian.dieckmann@astrazeneca.com

\section{KARGER} (1) 2013 S. Karger GmbH, Freibur 


\section{Afinitor ${ }^{\circledR}$ (Everolimus) beim fortgeschrittenen RCC: Beobachtungsstudie CHANGE zeigt mTTP von 7 Monaten}

Patienten mit fortgeschrittenem Nierenzellkarzinom (RCC), die während oder nach einer gegen VEGF (Vascular Endothelial Growth Factor) gerichteten Therapie progredient sind, profitieren auch unter Praxisbedingungen von Everolimus (Afinitor ${ }^{\circledR}$ ). Dies zeigt die seit Kurzem als Vollpublikation veröffentlichte 2. Interimsanalyse der nichtinterventionellen Studie (NIS) CHANGE (Charakterisierung von Afinitor $^{\circledR}$ nach gezielter Ersttherapie). In dieser wurden die Daten von 196 mit Everolimus behandelten Patienten (Gesamtpopulation) aus 79 deutschen Zentren dokumentiert, die zwischen August 2009 und September 2011 mindestens 3 Monate beobachtet wurden.

In der Interimsanalyse erreichten die Patienten der Effizienzpopulation* $(n=165)$ eine mediane Zeit bis zur Tumorprogression (mTTP) von 7,0 (95\%-KI 5,1-9,0) Monaten (Abb.1). Auch die Patienten, die vor Everolimus nur einen VEGFr-TKI (VEGF-Rezeptor-Tyrosinkinase-Inhibitor; $\mathrm{n}=121$ ) erhalten haben, erzielten eine mTTP von 7,1 (95\%KI 5,5-9,0) Monaten [1].

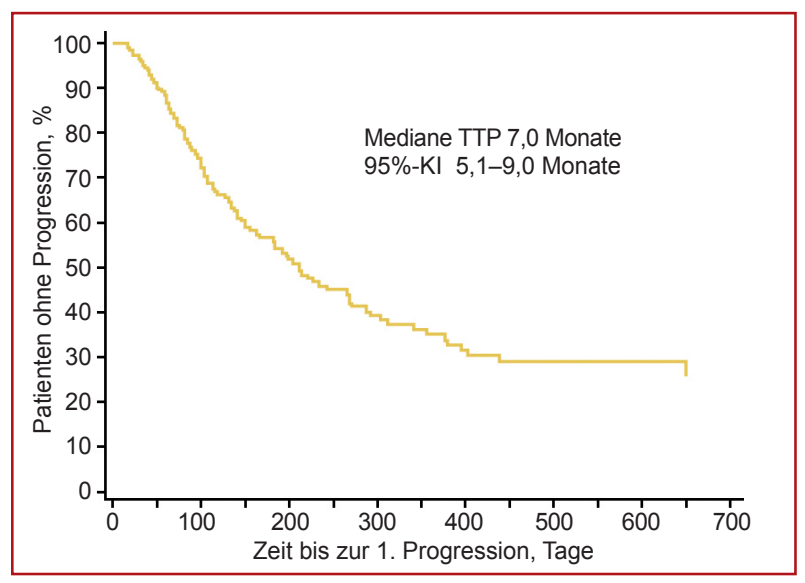

NIS CHANGE bestätigt Subgruppenanalyse der Zulassungsstudie RECORD-1

Damit bestätigen die Daten der Beobachtungsstudie CHANGE zur Wirksamkeit von Everolimus nach der 1. VEGFr-TKI-Therapie die Ergebnisse der geplanten Subgruppenanalyse der Zulassungsstudie RECORD (Renal Cell Cancer treatment with oral RAD001 given Daily)-1. In der von Calvo et al. vorabgeplanten Subgruppenanalyse konnte gezeigt werden, dass Patienten unter Everolimus, die mit nur einem TKI (Sunitinib oder Sorafenib) vorbehandelt wurden $(n=205)$, ein medianes progressionsfreies Überleben (PFS) von 5,4 Monaten erzielten im Vergleich zu 1,9 Monaten unter Placebo ( $n=103$; $p<0,001)$. Bei vorheriger Behandlung mit 2 TKIs lag das mediane PFS unter Everolimus $(\mathrm{n}=72$ ) lediglich bei 4,0 Monaten im Vergleich zu 1,8 Monaten unter Placebo $(\mathrm{n}=36 ; \mathrm{p}<0,001)[2]$.

Sicherheitsprofil vergleichbar mit der Zulassungsstudie

Das in der 2. Interimsanalyse der Beobachtungsstudie bei TKI-vorbehandelten Patienten aus dem Versorgungsalltag dokumentierte Sicherheitsprofil war ebenfalls mit dem in der RECORD-1-Studie [3] vergleichbar. Als häufigste unerwünschte Ereignisse

Abb. 1. Mediane Zeit bis zur Progression (TTP) in der Population für die Wirksamkeitsanalyse [1]. jeglichen Schweregrades traten in der Sicherheitspopulation** $\left.^{* *} \mathrm{n}=195\right)$ Dyspnoe $(14 \%)$, Anämie (13\%) und Schmerzen (9\%) auf. Obwohl bei $26 \%$ der Patienten eine Anpassung der Everolimus-Dosierung erforderlich war und bei $13 \%$ eine Therapieunterbrechung von median 16 (5-53) Tagen erfolgte, war die Therapie-Adhärenz mit ca. 97\% sehr hoch [1].

NIS CHANGE bildet Praxisalltag ab Die kurz nach der Zulassung von Everolimus begonnene NIS CHANGE untersucht erstmals systematisch die Wirksamkeit und Sicherheit dieses mTOR-Inhibitors beim fortgeschrittenen RCC in einem Real-Life-Setting. Mit einer mTTP von 7,0 Monaten, einem günstigen Sicherheitsprofil und einer Therapie-Adhärenz von ca. $97 \%$ bestätigt die 2 . Interimsanalyse der Beobachtungsstudie CHANGE das günstige Nutzen-Risiko-Profil von Everolimus bei Patienten mit fortgeschrittenem RCC, die zuvor einen TKI erhalten haben.

* Effizienzpopulation $(\mathrm{n}=165)$ : Alle Patienten der Sicherheitspopulation $^{* *}$, die weniger als 90 Tage nach Behandlungsbeginn mit Everolimus dokumentiert wurden und vorher einen TKI erhalten hatten oder einen 2. TKI für weniger als 1 Monat.

** Sicherheitspopulation ( $\mathrm{n}=195)$ : Alle Patienten mit mindestens einer dokumentierten Verordnung von Everolimus und einer dokumentierten Visite nach Beobachtungsbeginn.

\section{Referenzen \\ 1 Bergmann L et al.: Onkologie 2013;36:95-100 \\ 2 Calvo E et al.: Eur J Cancer 2012;48:333-339. \\ 3 Motzer RJ et al.: Cancer 2010;116:4256-4265.}

Weitere Informationen bei

Novartis Pharma GmbH

Yasmin Messer

Roonstraße 25, 90429 Nürnberg

yasmin.messer@novartis.com

\section{PharmaTicker+++ PharmaTicker+++ PharmaTicker+++ PharmaTicker+++}

Roche. An den seit Februar 2012 zugelassenen BRAF-Inhibitor Vemurafenib (Zelboraf ${ }^{\circledR}$ ) wurde in der jetzt veröffentlichten interdisziplinären S3-Leitlinie «Diagnostik, Therapie und Nachsorge des Melanoms» der höchste Empfehlungsgrad A vergeben. Demnach soll «bei BRAF-Inhibitor-sensitiver BRAF-Mutation eine Therapie mit einem BRAFInhibitor durchgeführt werden.» Diese Empfehlung wurde vor dem Hintergrund der beobachteten Wirksamkeit von Vemurafenib in der Zulassungsstudie BRIM-3 ausgesprochen (Evidenzlevel lb).

Roche Pharma AG

Ulla Satzger

ulla.satzger@roche.com
Eisai. Die beim SABCS vorgestellte 301-Studie liefert erstmals einen direkten Vergleich zwischen Eribulin (Halaven ${ }^{\circledR}$ ) und Capecitabin $\left(\right.$ Xeloda $\left.^{\circledR}\right)$. In den co-primären Studienendpunkten (OS, PFS) wurde kein signifikanter Unterschied erreicht. Jedoch zeigte sich ein Trend für ein verlängertes Gesamtüberleben (OS) unter Eribulin. Die 1-Jahres-Analyse ergab einen signifikanten OS-Vorteil mit Eribulin bei $64,4 \%$ gegenüber $58 \%$ bei Capecitabin. eickhoff kommunikation $\mathrm{GmbH}$ Mareike Trippe trippe@medizinmarketingservice.de
Bayer. Beim ASCO-GI-Kongress 2013 wurden neue Daten zum Krebsmedikament Regorafenib vorgestellt. Darunter sind neue Analysen aus der zulassungsrelevanten Phase-III-Studie CORRECT mit Regorafenib bei metastasiertem Darmkrebs sowie Ergebnisse einer Phase-I-Studie von Regorafenib bei hepatozellulärem Karzinom. Regorafenib wurde im September 2012 auf Basis der CORRECT-Ergebnisse von der Gesundheitsbehörde FDA (USA) unter dem Handelsnamen Stivarga $^{\circledR}$ zur Behandlung von mCRC zugelassen.

Bayer Pharma AG

Anna Koch / anna.koch@bayer.com

\section{KARGER}

(ㅇ) 2013 S. Karger GmbH, Freiburg 